\title{
Advances in the Diagnosis and Detection of Sickle Cell Anemia \\ Fernandes Q
}

National Center for Cancer Care and Research (NCCCR), Hamad Medical Corporation, Doha, Qatar

*Corresponding author: Fernandes Q, National Center for Cancer Care and Research (NCCCR), Hamad Medical Corporation, Doha, Qatar, Tel: (+974) 4439 5777; Email: queenie.fernandez@gmail.com

Received date: February 26, 2018; Accepted date: August 28, 2018; Published date: September 03, 2018

Copyright: () 2018 Fernandes Q. This is an open-access article distributed under the terms of the Creative Commons Attribution License, which permits unrestricted use, distribution, and reproduction in any medium, provided the original author and source are credited

\section{Abstract}

Sickle Cell Anemia (SCA) is a class of hemoglobinopathies that is distinguished by the mutation-induced expression of a sickle cell variant of hemoglobin $(\mathrm{HbS})$. This $\mathrm{HbS}$ variant triggers erythrocytes to take a characteristic sickled conformation. The complex physiopathology of the disease has led to the development and use of diverse diagnostic methodologies. This review attempts to classify the advancements in the diagnosis and detection of SCA traits.

Keywords: Sickle Cell Anemia; Erythrocytes; Neonatal diagnosis

\section{Introduction}

Traditional methods employed in the diagnosis of SCA involved the use of Hemoglobin Electrophoresis and High Performance Liquid Chromatography (HPLC). HPLC is an automated technique and hence is less laborious. Gradually focus shifted to Isoelectric Focussing (IEF) which offers a higher resolution and is more cost effective. However HPLC has the ability to differentiate between different $\mathrm{Hb}$ variants and hence is more reliable [1].

In a particular study using microarrays carried out by Cheng et al. [2] it was identified that although mature erythrocytes are enucleate, they still contain relatively small quantities of micro-RNAs (miRNAs) that help in regulating gene expression levels at the posttranscriptional level. Moreover the miRNA expression of the erythrocytes showing the sickle cell trait (HbAS) was completely different from that of the normal erythrocytes (HbAA). The diseased erythrocytes showed expression levels that closely resembled those of reticulocytes. Evidently this served as a biomarker for the diagnosis of SCA. These findings marked a milestone in the history of erythroid genetics; stressing on the use of microarrays in SCA diagnosis.

Diagnostic tools can be evaluated on the basis of whether they are high-throughput, have the capacity to differentiate between the different $\mathrm{Hb}$ variants or on the basis of being cost-effective and less labor-intensive.

\section{Prenatal diagnosis}

\section{Invasive prenatal diagnosis}

Conventional fetal sampling methods have always been invasive. These methods have shown a high success rate and are still practiced.

\section{Chorionic Villi sampling (CVS)}

This method is also called Trophoblast sampling and is usually carried out within 10-12 weeks of gestation. Here fetal material is made available through the chorion villi.
In order to carry out a diagnostic test for SCA sufficient fetal DNA can be isolated from approximately $20 \mathrm{mg}$ of sample [3]. The test is performed through the Allele Specific Oligonucleotide hybridization $(\mathrm{ASOH})$ method [4]. This method involves the generation of specific oligonucleotide probes containing the base sequences complementary to the mutation which is followed by subsequent hybridization with the denatured fetal DNA.

Currently commercial silica-membranes are available to carry out chorionic villus sampling.

\section{Amniocyte DNA Sampling}

Amniocyte sampling is carried out after 15 weeks of gestation. The fetal genetic material is isolated from the amniotic fluid using commercially available isolation kits. Amplification Refractory Mutation System (ARMS) have by far been the conventional method used to test fetal DNA obtained from these samples. This method is based on the use of specific labelled oligonucleotide primers complementary to the possible mutation in the fetal DNA. No amplification suggests that diagnosis is negative and vice-versa. Through this method, hundreds of fetal samples can be analyzed in a single diagnostic test.

\section{Non-Invasive prenatal diagnosis}

Although invasive-techniques have been a successful mode of fetal sampling, apart from being laborious, it also carries a small risk of fetal loss [4]. Tremendous work is now being put into newly emergent techniques of non-invasive diagnosis.

\section{Preimplantation genetics diagnosis (PGD)}

There has always been a certain physical and emotional discomfort surrounding the abortion of abnormal pregnancies. PGD is aimed at abolishing the need of abortion and its consequences by performing diagnosis before implantation.

The technique involves gamete collection from both parents followed by in-vitro fertilization of each of these gametes separately. This gives rise to a number of embryos for early diagnosis. Single cells are biopsied from the blastomer and tested for SCA mutation through DNA microarray detection. If the embryo has tested negative it is then 
transferred into the uterus of the mother for implantation. This technique enables the testing of hundreds of blastomers at a time and only a healthy blastomer is selected for embryo transfer.

\section{Free fetal DNA diagnosis}

Research has proven that fetal DNA circulates freely within the maternal blood. This free fetal DNA could be identified and isolated from the maternal blood by special techniques like micromanipulation $[4,5]$. Extracted free fetal DNA is then immobilized on a DNA microarray plate and hybridized with a probe specific to the mutation and diagnosis is carried out by suitable visualization.

\section{Neonatal Diagnosis}

When SCA has not been diagnosed at the prenatal level, it is essential to enable detection in new born infants to allow early commencement of treatment and comprehensive disease management. In a particular report Gaston et al. [6] stated that treatment of infants with penicillin prophylaxis considerably reduced mortality rate, thereby insisting on the implementation of neonatal screening programs. In 1987 the NIH Consensus Development Conference concluded that universal neonatal screening for SCA should be implemented so that penicillin prophylaxis treatment could be administered early.

In the diagnosis of SCA, it is essential to detect the different hemoglobin variants. In such cases, additional confirmatory tests are necessary. These tests are particularly important in neonates if a blood transfusion is received prior to diagnosis. Usually HPLC, IEF or hemoglobin electrophoresis is performed in order to carry out secondary tests [7].

\section{Dried blood spot sample (DBSS) screening}

Neonatal screening programs in many countries use Dried Blood Spot Sample (DBSS) for the diagnosis of SCA. DNA extracted from DBSS forms the target during DNA microarray diagnosis and is tested against a specific probe. Thus a large number of infants can be tested for SCA in a single test. Alternatively DNA from a single infant can be tested against a variety of mutation-specific oligonucleotide probes for a number of hemoglobinopathies apart from SCA. Thus a single diagnostic test could be used to identify a number of mutations.

However, neonatal SCA screening should preferably be done 2-3 months after birth as it is difficult to detect HbS quantities below $10 \%$. In infants a major fraction of the blood consists of fetal hemoglobin $(\mathrm{HbF})$ and hence screening should be done after $\mathrm{HbF}$ is replaced by adult hemoglobin [8].

\section{Adult Diagnosis}

\section{Split-oligonucleotide analysis systems}

Traditional methods used to confirm the genotype of hemoglobin in an individual was based on performing electrophoresis on cellulose acetate at a slightly basic $\mathrm{pH}$ [9]. Although this method is still widely in practice, it offers low sensitivity apart from being labor intensive. This method is now being replaced by novel diagnostic tools based on the principle of hybridization.

Since SCA is caused by a single point mutation, hybridization experiments using long oligonucleotides portray higher probabilities of a mismatch leading to a false-positive diagnosis. In order to tackle this problem, diagnosis based on DNA microarrays use a single oligonucleotide split into two as probes. Thus two or more probes bind to a single target DNA with a gap of few bases in-between (Figure 1).

Oligonucleotide probes are short nucleotide sequences labelled with non-radioactive reporter moieties. These probes usually display chemiluminisence or fluorescence in order to enable detection. The systems also enable quantification of nucleic acids and are hence powerful tools. The probes used, allows enhanced discrimination of mis-matches as compared to long nucleic acid probes and are also known to display a greater sensitivity towards Single Nucleotide Polymorphisms (SNPs). This phenomenon results due to a larger impact on the target site. Thus due to their superior specificity, they greatly reduce chances of false-positive signals.

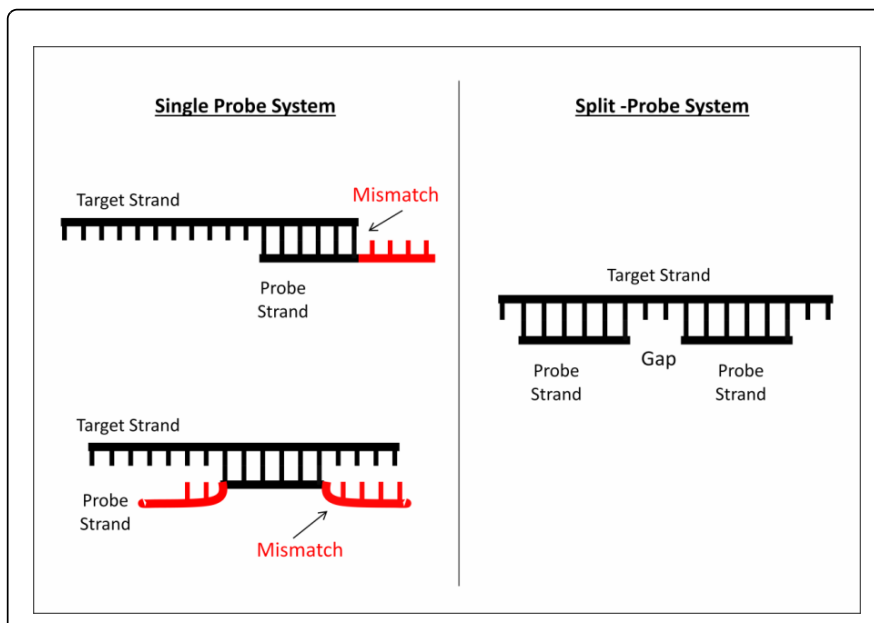

Figure 1: showing the use of split-probes to bind to the target site. Two probes bind to a single target with a gap of few base pairs in between.

The introduction of the split probe system has opened streams into newer and The introduction of the split probe system has opened streams into newer and improved diagnostic tools. Oligonucleotides are now being tagged with nanoparticles to be used in DNA bioassays. These probes have been tested and applied in microarrays, in homogenous reactions and in Surface Enhanced Raman Spectroscopy (SERS). In a particular study, split probes were coupled with gold nano-particles in order to enable distance-dependent detection [10]. A red color is displayed if the inter-particle distance is greater than the approximate particle diameter; otherwise a blue color becomes visible.

These particle bound probes are designed to hybridize at the target site in a head to tail manner enabling rapid detection without the need of sophisticated instrumentation.

\section{Conclusion}

The year 2010 marked a century since SCA was first detected in the blood in 1910 by James Herrick. A hundred years since then, researchers now are still striving to battle the disease and its associated clinical conditions. Evidently, early diagnosis leads to early treatment and better prognosis of the disease.

The incidence of SCA has dropped considerably. This decrease is attributed to the advancements in diagnostic techniques and the 
Citation: Fernandes Q (2018) Advances in the Diagnosis and Detection of Sickle Cell Anemia. J Blood Lymph 8: 222. doi: $10.4172 / 2165-7831.1000 .222$

Page 3 of 3

implementation of mutation screening programs. However, a crucial set back lies in the inadequate spread of awareness on diagnostic campaigns, especially in remote areas of Africa \& Asia. This remains to be a major issue contradicting the advancement in diagnostic technology. Most developing countries still cling on to traditional methods of diagnosis and treatment, thus hampering clinical progress.

\section{References}

1. Chapman CS (1999) Neonatal screening for hemoglobinopathies. Clin Lab Haematol 21: 229-234.

2. Chen SY, Wang Y, Telen MJ, Chi JT (2008) The Genomic Analysis of Erythrocyte microRNA Expression in Sickle Cell Diseases. PloS one.

3. Harteveld CL, Kleanthous M, Traeger-Synodinos J (2009) Prenatal diagnosis of hemoglobin disorders: Present and future strategies. Clin Biochem 42: 1767-1779.

4. McEwan A (2003) Pre-natal diagnosis of single gene disorders. Curr Obstet Gynaecol.
5. Tabor A, Philip J, Madsen M, Bang J, Obel EB, et al. (1986) Randomised controlled trial of genetic amniocentesis in 4606 low-risk women. Lancet 1: 1287-1293.

6. Gaston MH, Verter JI, Woods G, Pegelow C, Kelleher J, et al. (1986) Prophylaxis with oral penicillin in children with sickle cell anemia. N Engl J Med.

7. Reed W, Lane P, Lorey F, Bojanowski J, Glass M, et al. (2000) Sickle Cell Disease not identified by newborn screening because of prior transfusion. J Pediatr 136: 248-250.

8. Bhardwaj U, Zhang YH, McCabe ER (2003) Neonatal hemoglobinopathy screening: molecular genetic technologies. Mol Genet Metab

9. Akinyanju O, Disu RF, Akinde JA, Adewole TA, Otaigbe AI, et al. (1999) Initiation of Prenatal Diagnosis of Sickle-cell Disorders in Africa. Prenat Diagn 19: 299-304.

10. Elghanian R, Storhoff JJ, Mucic RC, Letsinger RL, Mirkin CA (1997) Selective colorimetric detection of polynucleotides based on the distancedependent optical properties of gold nanoparticles. Science 277: 1078-1081. 